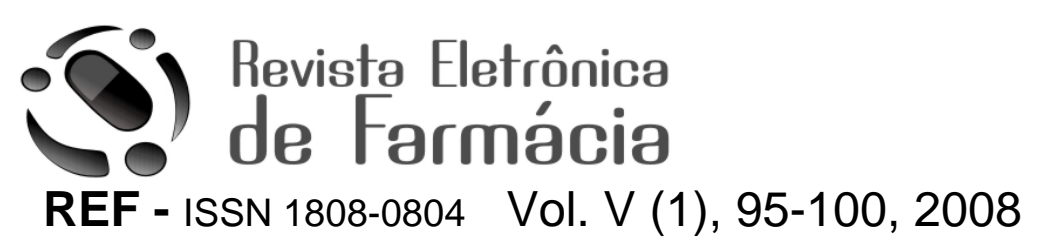

\title{
INCENTIVO A ASSISTENCIA FARMACEUTICA NA ATENÇÃO BÁSICA: UM PROJETO EM DISCUSSÃO
}

\author{
Incentive Pharmaceutical Care In Basic Atention: A Discussion Project \\ Yula de Lima Merola; ${ }^{1 *}$ Fábio de Souza Terra $;{ }^{2}$ Ana Maria Duarte Dias Costa ${ }^{3}$ \\ ${ }^{1}$ Secretaria Municipal de Saúde, Prefeitura Municipal de Poços de Caldas. \\ Rua Junqueiras $1963^{\circ}$ andar. 37701000 . Poços de Caldas-Mg, Brasil \\ 2,3 Docente da Pós Graduação da Universidade José do Rosário Vellano- Unifenas \\ Rua Rod. MG 179, Km 0 - Câmpus Universitário. 37130-000 Alfenas-MG
}

*autor para correspondência email: yula.merola@uol.com.br

Recebido em 09/07/2008 - Aceito em 31/07/2008

RESUMO: Segundo a OMS, o conceito de Atenção Farmacêutica (AF) é um compêndio de atividades, comportamentos, compromissos, inquietudes, valores éticos, funções, conhecimentos, responsabilidades e habilidades do farmacêutico na prestação da farmacoterapia, com o objetivo de alcançar resultados terapêuticos definidos na saúde e na qualidade de vida do paciente. Os elementos da Atenção Farmacêutica configuram uma atenção integral ao paciente, onde a prestação exige uma relação contínua e concordada entre os profissionais de saúde e o paciente. E para efetiva implementação da AF no SUS, a Política Nacional de Medicamentos (PNM) constitui-se parte fundamental para a implantação de ações capazes de promover a melhoria das condições da assistência à saúde da população. O seu objetivo primeiro é garantir a necessária segurança, eficácia e qualidade dos medicamentos, a promoção do uso racional e o acesso da população àqueles considerados essenciais, por envolver atividades de caráter abrangente e multiprofissional, que têm como finalidade à promoção do acesso a medicamentos de qualidade, assim como de seu uso racional. A importância de uma política de assistência farmacêutica é inquestionável, tendo como prioridade a reestruturação administrativa das áreas responsáveis pela política de medicamentos como fundamental para uma dinamização dos programas desenvolvidos. Apesar dos avanços obtidos, não se pode dizer que a assistência farmacêutica do SUS esteja totalmente resolvida, atendendo à demanda nos itens e quantidades desejados. Para a continuidade da ampliação do acesso aos medicamentos, será preciso da parte dos novos gestores da saúde um especial cuidado com os custos envolvidos. Para viabilizar uma política de assistência farmacêutica é preciso que seu financiamento esteja solidamente estruturado, uma vez que os valores envolvidos são expressivos e o mercado não é plenamente concorrencial. Para que o acesso aos medicamentos seja ampliado, é preciso que os instrumentos de monitoramento e intervenção no mercado sejam cada vez mais aprimorados.

Palavras-chave: Assistência Farmacêutica, Uso de Medicamentos, Política Nacional de Medicamentos

ABSTRACT: According to the OMS (World Health Organization), the concept of Pharmaceutical Care is a collection of activities, behavior, commitments, uncertainties, ethical values, functions, knowledge, responsibilities, and abilities of a pharmacist in providing pharmaceutical treatment with the objective of achieving therapeutical results, ultimately defined in the patient's health and quality of life. Elements of Pharmaceutical Care place the pharmacist's care integrally on the patient, in those cases where treatment requires a continuous relationship and agreement between health professionals and the patient. For an effective implementation of Pharmaceutical Care into the Brazilian National Health Service (named SUS), the National Drug Policy (PNM) is a fundamental component, made up of actions which are capable of encouraging the improvement of healthcare conditions within the population. The first objective is to guarantee the security, efficiency, and quality of medication required, encouraging drug utilization, and giving the population access to medications which are considered essential, involving far-reaching and multi-disciplinary activities with the goal of encouraging access to quality medication, as 
Merola, Y. R. et al./Revista Eletrônica de Farmácia Vol 5(1), 95-100, 2008.

well as their rational use. The importance of a pharmaceutical assistance policy is unquestionable; administrative restructuring in the areas responsible for medication policy is a fundamental priority in making the developed programs more dynamic. Despite the advances that have been made, the health service's pharmaceutical assistance cannot be said to be completely reformed; being still unable to meet the demand for items or the quantities required. In order to continue expanding access to medications, new healthcare managers will need to exercise special care with the costs involved. To make the pharmaceutical assistance policy viable, its financing must be solidly restructured since the values involved are large and the market is not completely competitive. Monitoring instruments and market intervention must be continually improved to expand access to medication.

KEYWORDS: Pharmaceutical Care; National Drug Policy; Drug Utilization

\section{INTRODUÇÃO}

O acesso a medicamentos de qualidade e o uso racional são preocupações antigas da Organização Mundial da Saúde (OMS). Na área de medicamentos, além do uso racional, a OMS, contribui para a qualidade e a segurança dos medicamentos, incluindo medicamentos tradicionais, e promovendo o acesso eqüitativo e sustentável a medicamentos essenciais, principalmente àqueles para os pobres e necessitados (WHO, 1997).

Dados recentes relatam que o mercado farmacêutico brasileiro é um dos cinco maiores do mundo. O perfil dos consumidores deste mercado demonstra que $15 \%$ da população com renda superior a dez salários mínimos consome $48 \%$ da produção destes medicamentos, enquanto que, mais da metade da população (51\%), com renda até quatro salários mínimos, é responsável pelo consumo de somente $16 \%$ dos medicamentos produzidos. Os 34\% que têm renda mensal entre 4 e 10 salários mínimos consomem $36 \%$ dos medicamentos do mercado (BRASIL, 1999; ROMANOLIEBER, 2000). Este fato evidencia claramente que, por razão sócio-econômico, o acesso à terapia medicamentosa não ocorre de forma igual na população, ficando comprometido para os milhões de brasileiros que têm baixa renda (FERRAES, 2000).

\section{CONCEITOS E AVALIAÇÃO DA ASSISTÊNCIA FARMACÊUTICA}

Segundo a Organização Mundial de Saúde (OMS), o conceito de Atenção Farmacêutica é um compêndio de atividades, comportamentos, compromissos, inquietudes, valores éticos, funções, conhecimentos, responsabilidades e habilidades do farmacêutico na prestação da farmacoterapia, com o objetivo de alcançar resultados terapêuticos definidos na saúde e na qualidade de vida do paciente. O termo Atenção Farmacêutica significa o processo pelo qual o farmacêutico atua com os profissionais e com o paciente na planificação, implementação e monitorização de uma farmacoterapêutica que produzirá resultados específicos. O aconselhamento ao paciente é um dos instrumentos essenciais para a realização da Atenção Farmacêutica, sendo imprescindível o desenvolvimento das habilidades de comunicação, para assegurar a boa relação farmacêutico - usuário (ADAMES, 1997).

A Atenção Farmacêutica baseia-se, justamente, na capacidade do farmacêutico de assumir novas responsabilidades relacionadas aos medicamentos e aos pacientes, através da realização de um acompanhamento sistemático e documentado com o consentimento dos mesmos. No processo de Atenção Farmacêutica o paciente é o principal beneficiário das ações do farmacêutico. Os elementos da Atenção Farmacêutica configuram uma atenção integral ao paciente, onde a prestação exige uma relação contínua e concordada entre os profissionais de saúde e o paciente. O farmacêutico deve utilizar seu critério clínico para determinar o nível de atenção que cada paciente necessita (FERRAES, 2000).

Dessa forma, a Assistência Farmacêutica engloba as ações de Atenção Farmacêutica quando se refere às ações específicas do profissional farmacêutico com o paciente buscando o atendimento de suas necessidades vinculadas ao uso de medicamentos. Tais pressupostos são reafirmados em eventos e documentos oficiais onde se expressa a necessidade de descentralizar a Assistência Farmacêutica a fim de possibilitar a proximidade do serviço ao paciente com a presença do farmacêutico para orientação e educação individualizada e garantir a qualidade da Atenção Farmacêutica (COSTA, 2007).

Para a OMS (1993) a Atenção Farmacêutica é fundamental para reduzir os gastos dos governos com a saúde pública, para desafogar a assistência médica, para melhorar a compreensão do uso adequado de drogas por parte dos paciente.

A assistência farmacêutica no SUS é composta por todas as etapas relacionadas ao medicamento: seleção, programação, aquisição, armazenamento, distribuição, controle da qualidade e utilização do medicamento (compreendendo nesta, a prescrição e dispensação) (WHO, 1997; BRASIL, 1999b). Estas atividades deverão favorecer a disponibilidade permanente de medicamentos conforme necessidades da população a serem identificadas com base em critérios epidemiológicos, tendo como finalidade fundamental de promover a farmacoterapia planejada, para encontrar os resultados definitivos que melhorem a qualidade de vida do paciente (ADAMES, 1997; BRASIL, 1999). 
Merola, Y. R. et al./Revista Eletrônica de Farmácia Vol 5(1), 95-100, 2008.

De acordo com a Portaria 3916 que apresenta a Política Nacional de Medicamentos, a Assistência Farmacêutica é definida como: "Grupo de atividades relacionadas com o medicamento, destinadas a apoiar as ações de saúde demandadas por uma comunidade. Envolve o abastecimento de medicamentos em todas e em cada uma de suas etapas constitutivas, a conservação e o controle de qualidade, a segurança e a eficácia Terapêutica dos medicamentos, o acompanhamento e a avaliação da utilização, a obtenção e a difusão de informação sobre medicamentos e a educação permanente dos profissionais de saúde, do paciente e da comunidade para assegurar o uso racional de medicamentos (BRASIL, 1999).

E para sua efetiva implementação da AF no SUS, a Política Nacional de Medicamentos (PNM) constitui-se em parte fundamental para a implantação de ações capazes de promover a melhoria das condições da assistência à saúde da população. O seu objetivo primeiro é garantir a necessária segurança, eficácia e qualidade dos medicamentos, a promoção do uso racional e o acesso da população àqueles considerados essenciais, sendo a reorientação da Assistência Farmacêutica uma das diretrizes fundamentais da Política Nacional de Medicamentos, por envolver atividades de caráter abrangente, multiprofissional e intersetorial, que têm como finalidade precípua à promoção do acesso a medicamentos de qualidade, assim como de seu uso racional; tendo como objeto de trabalho a organização das ações e serviços relacionados ao medicamento e voltadas ao usuário, a Assistência Farmacêutica deve ser abordada de forma que não fique restrita somente à aquisição e à distribuição de medicamentos (BRASIL, 2001). A PNM formaliza uma definição para a assistência farmacêutica ampla e integral que, envolve o abastecimento de medicamentos em todas e em cada uma de suas etapas constitutivas, a conservação e controle de qualidade, a segurança e a eficácia terapêutica dos medicamentos, o acompanhamento e a avaliação da utilização, a obtenção e a difusão de informação sobre medicamentos e a educação permanente dos profissionais de saúde, do paciente e da comunidade para assegurar o uso racional de medicamentos (BRASIL, 1999).

A Organização Mundial da Saúde (OMS) tem enfatizado a importância da formulação e implementação de uma PNM, no contexto SUS como ferramenta que permite conhecer o progresso da implementação da política, dando subsídios aos formuladores para rever as metas propostas, permitindo o aperfeiçoamento sistemático (BRASIL, 2002b). Um amplo e diverso número de atividades compõem a definição acima, estas são atividades multiprofissionais em várias etapas, voltadas, em seu conjunto, a integrar as ações de saúde.

Tem ocorrido forte consenso em tratar como componentes da assistência farmacêutica os processos de seleção, programação, aquisição, armazenamento, distribuição, e dispensação/utilização de medicamentos. A dispensação, um dos componentes do ciclo da Assistência Farmacêutica, é o ato farmacêutico de distribuir um ou mais medicamentos a um paciente, geralmente como resposta à apresentação de uma prescrição elaborada por um profissional autorizado. Neste ato o farmacêutico informa e orienta o paciente sobre o uso adequado do medicamento. São elementos importantes desta orientação, entre outros, a ênfase no cumprimento do regime de dosificação, a influência dos alimentos, a interação com outros medicamentos, o reconhecimento de reações adversas potenciais e as condições de conservação do produto (MARIN et al., 2003). O procedimento da dispensação deve assegurar que o medicamento de boa qualidade seja entregue ao paciente certo, na dose prescrita, na quantidade adequada; que sejam fornecidas informações suficientes para o uso correto e que seja embalado de forma a preservar a qualidade do produto (ZANINI et al., 1985). No momento do atendimento é relevante considerar as especificidades individuais do paciente, isto é, cada paciente é único e portanto apresenta características e necessidades específicas. E é neste momento também, que se tem a possibilidade de pontuar, retificar e/ ou minimizar prováveis riscos ao paciente relacionados à terapêutica medicamentosa, ainda dentro do sistema de saúde (IVAMA, 2001). Os aspectos identificados quanto à qualidade em saúde devem ser aqui considerados. Assim, da mesma forma que para o cuidado em saúde de forma geral, a relação entre qualidade e custo do cuidado e qualidade e quantidade de atenção, deve ser examinada com cuidado. Da mesma maneira, a qualidade técnica dos serviços ou produtos oferecidos devem ser acompanhadas de relação interpessoal adequada, como fatores importantes e interligados, para alcançar os resultados que podem ser a cura da doença, eliminação ou redução da sintomatologia, interrupção ou diminuição da progressão da doença ou prevenção da doença ou da sintomatologia (SANTOS, 2000). A Assistência Farmacêutica deve ser abordada como componente do cuidado à saúde, que tem o medicamento como um importante instrumento para o aumento da resolutividade do atendimento ao usuário (OPAS, 2005).

\section{ASSISTÊNCIA FARMACÊUTICA NA ATENÇÃO BÁSICA DE SAÚDE}

Com a municipalização, a questão dos medicamentos essenciais destinados à atenção básica passa a ser prerrogativa e responsabilidade municipal. Assim, em relação à AF, a Portaria 176 publicada pelo MS em março de 1999, estabelece critérios e requisitos para a qualificação dos municípios e estados ao Incentivo à Assistência Farmacêutica Básica e define valores a serem transferidos (BRASIL, 1990 b; CARVALHO, 1995; BRASIL, 1999 a).

Diante do exposto, uma das responsabilidades do gestor municipal no âmbito do SUS em relação à Atenção Farmacêutica (AF) é garantir o suprimento adequado de medicamentos essenciais a sua população, uma vez que recebe o incentivo destinado à assistência farmacêutica básica (BERCKER, 1985). Também são previstas responsabilidades para os gestores nas três esferas do governo (Municipal, Estadual e Federal). Assim, os 
Merola, Y. R. et al./Revista Eletrônica de Farmácia Vol 5(1), 95-100, 2008.

gestores do SUS são direcionados a procurar reorientar a AF, concentrando esforços e atuando em parcerias para assegurar o acesso da população a medicamentos seguros, eficazes e de qualidade, ao menor custo possível (BRASIL, 1999). Em relação às responsabilidades de que trata a PNM, ao governo federal gestores estaduais caberá, por exemplo, apoiar a organização de consórcios destinados à prestação da assistência farmacêutica ou estimular a inclusão deste tipo de assistência como objeto de consórcios de saúde. Já o gestor municipal poderá associar-se a outros municípios, por intermédio da organização de consórcios, tendo em vista a execução da assistência farmacêutica (BRASIL, 1999).

Bermudez et al. (1999) falam do desafio de inserir a AF na assistência à saúde e consideram-na uma das áreas mais críticas da assistência à saúde. Para o autor, a falta de medicamentos compromete a imagem dos serviços e pode ocasionar internações desnecessárias onerando ainda mais o sistema de saúde. Sabe-se que o uso irracional, desnecessário e inadequado do medicamento também gera aumento na demanda. Conforme Zanini et al. (1985) "compliance" é o termo utilizado para definir o nível de aceitação, cooperação e cumprimento das instruções por parte do paciente em relação ao tratamento médico recebido.

Neste prisma, a orientação farmacêutica sobre a utilização correta do medicamento pode ser considerada uma estratégia de qualidade para adesão ao tratamento e para uso racional do medicamento (FERRAES, 2002). A premissa básica para o MS é a descentralização dos medicamentos considerados essenciais, a aquisição e distribuição destes passam a ser responsabilidade dos municípios e sob a coordenação dos estados. Contudo, a reorientação proposta pelo MS não se restringirá só à aquisição e distribuição de medicamentos, mas também às demais etapas da AF (BRASIL, 1999).

Segundo Ivama (2001), no Brasil, vários projetos estão sendo implementados no sentido de modificar o objeto de trabalho do farmacêutico, priorizando o paciente e não mais o medicamento como fim em si mesmo. Menciona ainda que a promulgação da Portaria $n^{\circ}$. 3.916/GM de 30/10/1998 que dispõe sobre a Política Nacional de Medicamentos , a Lei 9.797/1999 como parte das estratégias adotadas pelo governo para racionalizar a utilização de medicamentos no país podem ser consideradas como condições propícias para o desenvolvimento de estratégias de educação continuada e permanente e a implementação de políticas para a formação de recursos humanos em Farmácia que contemple a prática da Atenção Farmacêutica de forma sistemática e integral no país (BRASIL, 1990b, BRASIL, 1999).

A proposta do Ministério da Saúde para a reorientação do modelo de AF, a partir da Política Nacional de Medicamentos tem como fundamentos: a descentralização da gestão; a promoção do uso racional de medicamentos; a otimização e eficácia na distribuição no setor público; e estímulo a iniciativas que conduzam a redução nos preços dos produtos. Além destas diretrizes, destacam-se ainda como prioridades: a adoção da RENAME, regulamentação sanitária de medicamentos, promoção da produção de medicamentos e desenvolvimento e capacitação de recursos humanos nos diversos setores e esferas envolvidos, permitindo acesso à informação e educação continuada (BRASIL, 1999).

O uso racional de medicamento segundo a PNM é o processo que compreende a prescrição apropriada; a disponibilidade oportuna e a preços acessíveis; a dispensação em condições adequadas; e o consumo nas doses indicadas, nos intervalos definidos e no período de tempo indicado de medicamentos eficazes, seguros e de qualidade (BRASIL, 1999).

As Políticas Públicas configuram decisões de caráter geral que apontam as linhas estratégicas de atuação de uma determinada gestão. A Política Nacional de Medicamentos do Brasil tem como propósito primeiro garantir a necessária segurança, eficácia e qualidade dos medicamentos, a promoção do uso racional e o acesso da população àqueles considerados essenciais, tendo por base os princípios e diretrizes do Sistema Único de Saúde brasileiro (BRASIL, 2002). Muitos países têm desenvolvido e implementado política nacional de medicamentos. Outros têm se direcionado no sentido de melhorias pontuais da qualidade da assistência farmacêutica. Contudo, ambos os processos requerem uma aproximação sistemática para a avaliação de políticas farmacêuticas. Em virtude disto, surge a necessidade da elaboração de instrumentos e desenvolvimento de metodologias que possibilitem um efetivo monitoramento da implementação da Política Nacional de Medicamentos e seus componentes, permitindo avaliação de desempenho e revisão das prioridades (OPAS, 1992).

\section{CONCLUSÕES}

Atenção Farmacêutica é uma filosofia que promoveu no mundo inteiro a reformulação da prática profissional em que o farmacêutico assume efetivamente compromissos com o paciente prestando-lhe serviços dentro das farmácias, públicas ou hospitalares, que podem melhorar sua qualidade de vida. Esses serviços podem ajudar a reverter os índices negativos de saúde da população (DUPIN et al, 1997).

A importância de uma política de assistência farmacêutica é inquestionável, tendo como prioridade a reestruturação administrativa das áreas responsáveis pela política de medicamentos como fundamental para uma dinamização dos programas desenvolvidos. A partir dessa reestruturação foi possível o aprofundamento da descentralização; o aperfeiçoamento dos processos de aquisição centralizada e a intensificação da intervenção e monitoramento do mercado. Isso resultou em um maior acesso da população e em novos medicamentos sendo incorporados aos programas de distribuição gratuita. Apesar dos avanços obtidos, não se pode dizer que a 
Merola, Y. R. et al./Revista Eletrônica de Farmácia Vol 5(1), 95-100, 2008.

assistência farmacêutica do SUS esteja totalmente resolvida, atendendo à demanda nos itens e quantidades desejados. Para a continuidade da ampliação do acesso aos medicamentos, será preciso da parte dos novos gestores da saúde um especial cuidado com os custos envolvidos. Para viabilizar uma política de assistência farmacêutica é preciso que seu financiamento esteja solidamente estruturado, uma vez que os valores envolvidos são expressivos e o mercado não é plenamente concorrencial. Para que o acesso aos medicamentos seja ampliado, é preciso que os instrumentos de monitoramento e intervenção no mercado sejam cada vez mais aprimorados (NEGRI, 2002).

\section{REFERÊNCIAS BIBLIOGRÁFICAS}

ADAMES, L. A. B. Medicamentos essenciais: gestão nos serviços públicos de saúde fundamentados no quadro nosológico. Divulgação em Saúde para Debate, CEBES, n.18, p. 34-42, jun. 1997.

BERMUDEZ, J.A.Z.; BONFIM, J. R. A. (org.). Medicamentos e Reforma do Setor Saúde. São Paulo: Hucitec/Sobravime, p. 236, 1999.

BRASIL. Ministério da Saúde. Portaria no. 176 de 8 mar. 99. Estabelece critérios e requisitos para a qualificação dos Municípios e Estados ao Incentivo à Assistência Farmacêutica Básica e defines valores a serem transferidos. Mar., 1999.

BRASIL, Ministério da Saúde. Secretaria de Políticas de Saúde. Assessoria de Assistência Farmacêutica. Como Gerenciar bem o incentivo à assistência farmacêutica básica. Brasília, p.27, 2000a.

BRASIL. Ministério da Saúde. Secretaria de Políticas de Saúde. Assessoria de Assistência Farmacêutica. Seleção de medicamentos. Brasília, Material fornecido na Oficina de Assistência Farmacêutica, 5 a 9/06/2000 em Curitiba $-\mathrm{PR}, 2000 \mathrm{~b}$

BRASIL. Ministério da Saúde. Secretaria de Políticas de Saúde. Departamento de Atenção Básica. Gerência Técnica de Assistência Farmacêutica. Incentivo à Assistência Farmacêutica Básica: o que é e como funciona. Brasília - DF, abril, p 25, 2001.

BRASIL, MINISTERIO DA SAÚDE, CONSELHO NACIONAL DE SAUDE. O Desenvolvimento do Sistema Único de Saúde: avanços, desafios e reafirmação dos seus princípios e diretrizes. Ministério da Saúde, Brasília. 2002b BRASIL. Ministério da Saúde. Secretaria de Políticas de Saúde. Departamento de Atenção Básica. Gerência Técnica de Assistência Farmacêutica. Assistência farmacêutica na atenção básica: instruções técnicas para sua organização. Brasília DF, abril, 113p, 2002a.

CARVALHO, G. I.; SANTOS, L. Comentários à Lei Orgânica de Saúde (Lei 8.080/90 e Lei 8.142/90): Sistema Único de Saúde. Ed. Hucitec: São Paulo, p 290, 1995.

CASTRO, O.C.G.S. (org,); MOSEQUI G.B.G.; PEIXOTO M.A.P.; CASTILHO S.R. \& LUIZA V.L., Estudos de utilização de medicamentos: noções básicas. Rio de Janeiro, FIOCRUZ, 2000.

COSENDEY, M.A.E. et al. Assistência Farmacêutica na atenção básica de saúde: a experiência de três estados brasileiros. Cadernos de Saúde Pública, 16(1): p. 171-182 2000.

COSTA, EULA M. Encontro Farmacoterapêutico. Revista Eletrônica de Farmácia. Vol. IV (1), 27-31, 2007.

DUPIM, J. A. A.; RIGHI, R. E. Medicamentos essenciais nos sistemas locais de saúde. In: BONFIM, J. R. A.; MERCUCCI, V. L. (org.). A Construção da Política de Medicamentos. São Paulo: Hucitec/Sobravime, p.138 - 154 , 1997.

FERRAES, A. M. B. Determinantes que impactam a atuação do Farmacêutico na Farmácia. Olho Mágico. Londrina, v.9, n.1, p. 151, jan./abr., 2002.

FERRAES, A. M. B. O profissional de farmácia: a busca de qualidade na atuação. In: Congresso Brasileiro de Saúde Coletiva, 7, Salvador. Livro de Resumos... Salvador: ABRASCO, v.5, suplemento, p. 134, 2000.

GOLDBAUM, M. Epidemiologia e serviços de saúde. Cadernos de Saúde Pública, Rio de Janeiro, v. 12 (suplemento 2), p. 95-98, 1996. 
Merola, Y. R. et al./Revista Eletrônica de Farmácia Vol 5(1), 95-100, 2008.

IVAMA, A. M (coord.). Delineamentos de estratégias de educação permanente de recursos humanos em farmácia. Projeto de pesquisa. Londrina, p 10, 2001.

MARIN, N. et al. Assistência Farmacêutica para gerentes municipais. OPAS/OMS, 2003.

NEGRI, BARJAS. Política Federal de Assistência Farmacêutica: 1990-2002. Sério B, Textos básicos de saúde. Editora MS, Brasília, 2002.

ORGANIZAÇÃO PAN-AMERICANA DA SAÚDE-OPAS. Guia para el diagnostico de farmacias y servicios farmacêuticos em las unidades de salud. Programa Regional de Medicamentos Essenciales, 1992.

ORGANIZACIÓN MUNDIAL DE LA SALUD. Organización Panamericana de la Salud. El papel del farmacéutico en el sistema de atención de salud. Buenas Prácticas de Farmacia. (Informe de la reunión de la OMS, Tokio, Japón, 31 de agosto al 3 de setiembro de 1993), 1993.

ROMANOLIEBER, N. S. Política de medicamentos. O Mundo da Saúde. Ed. São Camilo, ano 24, v. 24, n.1, p. 4550, jan/fev., 2000.

SANTOS, J. S. Atenção farmacêutica no Brasil. Pharmácia Brasileira, Brasília, v. 3, n. 19, p. 27-29, mar./abr. 2000. SECRETARIA DE POLÍTICAS DE SAÚDE. Política Nacional de Medicamentos. Rev. de Saúde Pública; 34 (2): $206-9,2000$.

WHO (Word Health Organization). The use of essential drugs. WHO Technical Report Series 867. Geneva: WHO, 1997.

ZANINI, C. A.; SANCHEZ, L. C.; PAULO, L. G. Assistência farmacêutica. In: ZANINI \& OGA. Farmacologia aplicada: uso racional do medicamento. 3. ed. São Paulo: Atheneu, cap. 83, p. 681-693, 1985. 